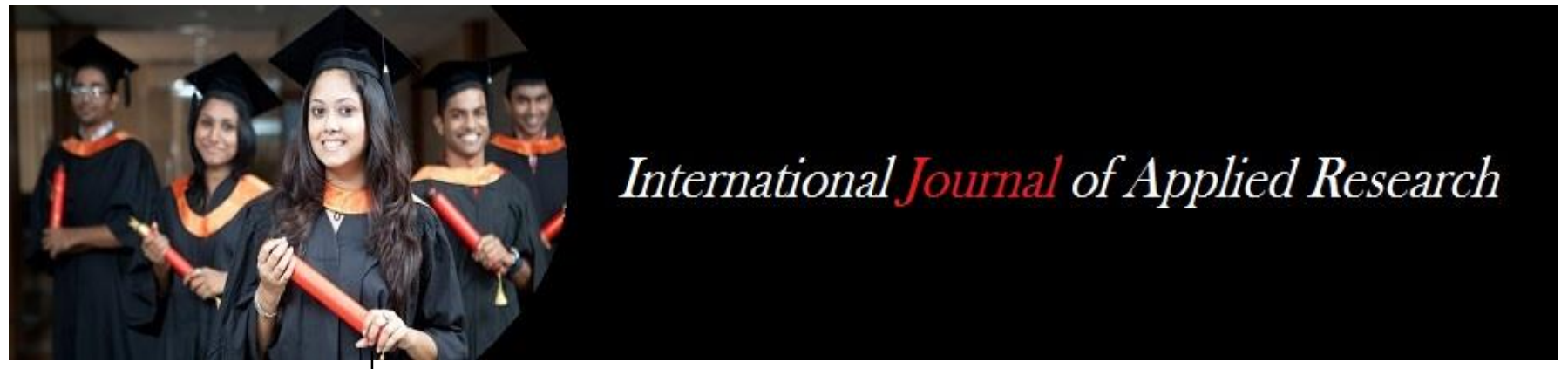

ISSN Print: 2394-7500 ISSN Online: $2394-5869$ Impact Factor: 8.4 IJAR 2021; 7(9): 268-270 www.allresearchjournal.com Received: 16-07-2021 Accepted: 22-08-2021

Sameer Ahmad Dar M.Sc., Department of Medical Surgical Nursing and Gastroenterology Nursing, Tutor, Government College of Nursing, Baghi Dilawar Khan, Srinagar, Jammu and Kashmir, India
Corresponding Author: Sameer Ahmad Dar M.Sc., Department of Medical Surgical Nursing and Gastroenterology Nursing, Tutor, Government College of Nursing, Baghi Dilawar Khan, Srinagar, Jammu and Kashmir, India

\section{Role of nurses in infection prevention and control in health care setting}

\section{Sameer Ahmad Dar}

DOI: https://doi.org/10.22271/allresearch.2021.v7.i9d.8994

\begin{abstract}
Infection prevention and control (IPC) is a practical, evidence-based approach which prevents patients and health workers from being harmed by avoidable infection and as a result of antimicrobial resistance. No one should catch an infection while receiving health care, yet, these infections can spread through outbreaks and many regular care practices, affecting hundreds of millions of people across the world every year. Infection prevention and control is one of the first topics introduced in nursing programs, yet its main tenets are often lost or forgotten.
\end{abstract}

Keywords: Role, nurses, infection, prevention, control, health

\section{Introduction}

Germs are a part of everyday life and are found in our air, soil, water, and in and on our bodies. Some germs are helpful, others are harmful. Many germs live in and on our bodies without causing harm and some even help us to stay healthy. Only a small portion of germs are known to cause infection. An infection happens when your body's immune system is unable to fight off bacteria, viruses, and other pathogens. A pathogen, commonly called a germ, causes illness. Your immune system is your body's way of fighting pathogens. It is a process that involves cells, organs, and proteins. When your immune system is working properly, white blood cells destroy harmful germs. When it is weak, your white blood cells have a harder time fighting infection. Hospital-acquired infections, also known as healthcareassociated infections (HAI), are nosocomial acquired infections that are typically not present or might be incubating at the time of admission. These infections are usually acquired after hospitalization and manifest 48 hours after admission to the hospital. Transmission of pathogens in a health care environment is complex and can occur through direct contact with the healthcare workers or the surrounding contaminated environment.

\section{What is Infection Control?}

Infection control is the practical discipline of preventing infections acquired in healthcare settings. Akin to a public health practice, infection control is an essential process of every healthcare organization. It addresses factors related to the spread of infections among patients, among staff, and between patients and staff. This includes preventive measures such as hand washing, cleaning, disinfecting, sterilizing, and vaccinating. Other aspects include monitoring and managing outbreaks of infection and investigating their causes.

\section{What is an Infection Control Nurse?}

An infection control nurse is a registered nurse (RN) who implements best practices for halting the spread of viruses and bacteria and delivers top care to patients who have contracted infectious diseases. In this profession, it is critical to have strong attention to detail, the ability to work well under pressure, and excellent communication skills. Infection control nurses work not only with patients and physicians but also with scientists, public health experts, and government agencies to protect the health of individuals and the public. Clostridioides difficile (C. diff), Staphylococcus aureus (staph), Klebsiella, and Escherichia 
Escherichia coli $(E$. coli) are the most common pathogens causing hospital-acquired infections. Some responsibilities of infection control nurses include:

- Gathering and analyzing infection data to make evidence-based decisions.

- Educating medical and public health professionals on infection prevention protocols to facilitate emergency preparedness.

- Isolating and treating infected individuals to contain the spread of infectious diseases.

- Assisting with the development of action plans in case of a community or hospital outbreak to minimize the potentially devastating impact.

- Collaborating with government agencies such as the CDC (Centers for Disease Control and Prevention) to ensure that infection control practices are implemented and enforced.

- Studying pathogens to determine origin in order to prevent future outbreaks.

- Assisting scientists and physicians with developing treatments and vaccines to ensure the health and safety of patients and the community.

\section{Role of Nurses in health care setting to control and prevent infection}

As a nurse, you must know how to protect yourself and your patients from exposure to harmful pathogens by understanding your organization's infection control policies and following them. These include standard precautions (hand hygiene, PPE, injection safety, environmental cleaning, and respiratory hygiene/cough etiquette) and transmission-based precautions (contact, droplet, and airborne).

Standard precautions: are guidelines that were established to break the chain of infection and reduce the risk of pathogen transmission in hospitals. Standard precautions apply to blood and body fluids, secretions and excretions (except sweat) and mucous membranes. Following standard precautions not only protects patients, but also healthcare workers.

Hand hygiene: is the number one weapon in preventing the spread of microorganisms and includes alcohol-based hand rubs and hand washing with soap and water. Alcohol-based hand rubs containing $60 \%$ to $95 \%$ alcohol are the preferred method for decontaminating hands, except when hands are visibly soiled or when a patient has infectious diarrhea. $C$. difficile and norovirus aren't affected by alcohol-based hand rubs; soap and water should be used in suspected or confirmed cases of infectious diarrhea.

Hand hygiene should be performed before and after contact with a patient; immediately after touching blood, body fluids, mucous membranes, or contaminated items (even when gloves are worn during contact); and immediately after removing gloves. Hand hygiene should also be performed when moving from contaminated body sites to clean body sites during patient care, before eating, after using the restroom, and after handling equipment in the vicinity of the patient. In addition to maintaining strict hand hygiene practices, patients and their family members should also be taught the importance of washing their hands.

The CDC recommends scrubbing hands for at least 20 seconds, using soap, water, and friction, and paying special attention to the areas between fingers, the backs of hands, underneath fingernails, and the thumbs. Alcohol-based hand rubs should be rubbed into all surfaces of the hands until dry.

PPE: (Personal Protective Equipment) includes gloves, gowns, masks, respirators, and eyewear that create barriers to protect skin, clothing, mucous membranes, and the respiratory tract from infectious organisms. The item selected depends on the infectious agent, the type of interaction, and the method of microorganism transmission. Gloves should be worn when touching blood, body fluids, mucous membranes, and contaminated items, and for any activities involving vascular access. A face shield or mask and goggles should be worn if you anticipate a splash or spray of blood or body fluids that might come in contact with your nose, eyes, or mouth. If you expect your skin or clothing might be exposed to body fluids or blood, wear a gown. Knowing how to put on and remove PPE can help prevent cross-contamination (see Donning and doffing $P P E$ ). To promote injection safety, gloves should be worn when administering injections. Puncture-proof disposal systems are recommended to dispose of uncapped needles and sharps. Never recap needles following administration of medication to reduce your risk of being stuck with an unclean needle. You should engage a needle safety device immediately after performing an injection.

Environmental cleaning: includes medical equipment and environmental surfaces. Any reusable equipment, including stethoscopes, bandage scissors, and hemostats, that's used on multiple patients must be cleaned between each patient contact, following organizational policy, with a broadspectrum antimicrobial agent such as chlorhexidine-a commonly used antimicrobial agent for disinfecting topical and hard surfaces in healthcare agencies. It's effective against Gram-positive and Gram-negative bacteria and fungi. Nursing staff should work closely with environmental services to ensure that rooms are thoroughly cleaned and disinfected between patients to prevent the spread of infection through inanimate objects.

Respiratory hygiene and cough etiquette: are infection control measures that should be implemented when contact is made with a patient who might have an upper respiratory infection. Patients with signs and symptoms of a respiratory infection should be taught to cover their mouth and nose with a tissue when coughing or sneezing and dispose of the tissue in the nearest trash container as soon as possible. These patients should also perform hand hygiene with alcohol-based rubs, soap and water, or an antiseptic hand wash after being exposed to respiratory secretions or contaminated materials or objects. Healthcare facilities should ensure adequate and readily accessible supplies of tissues and hand hygiene stations. Visual alerts should be posted in facility entrances to remind patients and visitors to inform healthcare professionals of respiratory signs and symptoms. Patients with respiratory symptoms should be masked to contain respiratory secretions.

Transmission-based precautions: Use transmission-based precautions in addition to standard precautions when the standard precautions aren't enough to protect you from communicable disease transmission. There are three types of 
transmission-based precautions: contact, droplet, and airborne.

Contact precautions: Are used in addition to standard precautions when caring for patients with known or suspected diseases that are spread by direct or indirect contact. Contact precautions include gloving and gowning when in contact with the patient, objects, and surfaces within the patient's environment. All reusable items should be cleaned and disinfected according to organizational policy, and disposable items should be thrown away immediately after being used.

Droplet precautions: Require the use of a surgical mask in addition to standard precautions when you're within $3 \mathrm{ft}(6 \mathrm{ft}$ for smallpox) of a patient known to have or suspected of having a disease spread by droplets. These include influenza, pertussis, and meningococcal disease. Healthcare personnel should observe droplet precautions when examining a patient with respiratory symptoms, especially if the patient has a fever. These precautions should remain in effect until it's determined that the symptoms aren't caused by an infection that requires droplet precautions.

Airborne precautions: Are used in addition to standard precautions when in contact with patients with known or suspected diseases spread by fine particles transmitted by air currents, such as tuberculosis, measles, and severe acute respiratory syndrome. You must wear a National Institute for Occupational Safety and Health certified, fit-tested N-95 respirator just before entry into an area shared with a patient suspected or known to have one of these diseases. Because there are several sizes of N-95 respirator, healthcare personnel must be fit tested according to organizational policy or at least every 2 years to be sure you're using the correct size. If eye protection is needed, wear goggles or a face shield during all contact with the patient, not just if you predict splashes or sprays.

\section{Conclusion}

Infection prevention and control is the responsibility of all health professionals. Nurses and midwives play a central role in breaking the chain of infection: they are in contact with people all the time and therefore, have the opportunity to prevent infections at every contact and intervention. Almost half the health and social care workforce is made up of nurses or midwives and can therefore have a significant impact on infection prevention. By understanding the chain of infection, the portals of exit and entry, and the modes of transmission, nurses and midwives are in a unique position to influence breaking the chain of infection.

\section{References}

1. Infection Prevention and Control by WHO. Available at: www.who.int/health-topics/infection-preventionand-control

2. Centers for Diseases Control and Prevention. Available at www.cdc.gov/infectioncontrol

3. Shaw K. Prevention by breaking the chain of infection. Nursing Times 2016;112(39, 40):12-14.

4. Patterson, Chris E, Basic Principles of Infection Control Nursing Made Incredibly Easy! 2015;13(3):28-37
5. Alberto F Monegro, Vijayadershan Muppidi, Hariharan Regunath. Hospital Acquired Infections. Available at: https://www.ncbi.nlm.nih.gov

6. What is the Role of an Infection Control Nurse? Available at: https://www.usa.edu/blog/infectioncontrol-nurse/

7. Infection Prevention and Control," Wikipedia: https://en.wikipedia.org/wiki/Infection_prevention_and _control 